\section{$\underset{\substack{\text { hommes } \\ \text { \& migrations }}}{ }$}

\section{Hommes \& migrations}

Revue française de référence sur les dynamiques

migratoires

$1279 \mid 2009$

L'Afrique en mouvement

\title{
Les migrations internes au continent africain
}

\section{Christophe Daum et Isaïe Dougnon}

\section{CpenEdition \\ Journals}

\section{Édition électronique}

URL : http://journals.openedition.org/hommesmigrations/280

DOI : 10.4000/hommesmigrations. 280

ISSN : 2262-3353

\section{Éditeur}

Musée national de l'histoire de l'immigration

Édition imprimée

Date de publication : 1 mai 2009

Pagination : 6-11

ISSN : 1142-852X

Référence électronique

Christophe Daum et Isaïe Dougnon, "Les migrations internes au continent africain », Hommes \& migrations [En ligne], 1279 | 2009, mis en ligne le 29 mai 2013, consulté le 10 décembre 2020. URL http://journals.openedition.org/hommesmigrations/280 ; DOl : https://doi.org/10.4000/ hommesmigrations.280 


\section{Les migrations internes au continent africain}

Par Christophe Daum et Isaïe Dougnon, Coordinateurs du dossier

Le présent dossier de la revue Hommes et Migrations rend compte de différentes recherches portant sur les migrations internes à l'Afrique Noire. On l'oublie souvent en Europe, mais la majorité des migrants demeure à l'intérieur même du continent. Soixante-dix millions d'Africains ont émigré, quittant leur pays pour s'installer durablement dans un autre. Et, à suivre les indicateurs fournis par l'OCDE ${ }^{(1)}$, ils ne sont qu'un peu plus d'un million et demi à être établis dans l'un des pays membres en 2005. Soit 2,6 pour cent du total des émigrés africains, et 2,63 pour cent ${ }^{(2)}$ du total des immigrés comptabilisés dans les pays de l'OCDE. Pourtant, la médiatisation des événements de Ceuta et Melilla en 2005, des naufrages de pirogues et les nombreuses disparitions qu'ils entrainent, ou encore les exigences de l'Europe négociant avec les pays d'origine ou dits de transit en vue de la "réadmission des immigrés clandestins" occultent les chiffres. Ces mêmes chiffres sont passés sous silence, voire dans le pire des cas manipulés et l'instrumentalisation des opinions publiques qui en découle concourt à mettre à mal les droits de l'Homme, dans un même mouvement de stigmatisation des migrants africains. En fait, "l'invasion" des pays européens par ces migrants n'est qu'un "mythe", pour reprendre le titre d'un récent rapport de Hans de Hass ${ }^{(3)}$ sur les migrations africaines vers l'Europe. Les "Subsahariens migrent peu vers l'Europe", ainsi que le montrent Beauchemin et Lessault dans le numéro de janvier 2009 de la revue Population et sociétés. La réalité des migrations africaines se joue donc principalement sur le continent, avec autant de trajectoires collectives et singulières qu'il y a de contextes de départ ou d'arrivée, à l'instar de ce continent trop souvent présenté comme un tout homogène alors qu'il est multiple. Les articles qui suivent présentent quelques une de ces dynamiques, à partir de recherches souvent originales et récentes.

Une première série d'articles porte sur des migrations rurales en Afrique de l'ouest, situations caractérisées par le dynamisme des populations concernées et qui montrent leur capacité d'adaptation aux contextes des pays d'installation. 
Une seconde série rappelle que les migrations en Afrique sont souvent, malheureusement, situées dans des contextes de crise. L'aggravation de la conjoncture tend parfois à exacerber les contradictions sociales et ces crises entraînent des mouvements de population ou durcissent les conditions de vie des immigrés.

- Daouda Gary-Tounkara retrace l'histoire des réseaux migratoires maliens, entre l'après-guerre et les Indépendances. Il montre comment ce tournant historique a favorisé l'émigration vers la Côte d'Ivoire, notamment autour de l'économie de plantation. À partir de ce premier espace migratoire, les réseaux vont se déployer vers d'autres pays du continent.

- Salif Togola rend compte de ses recherches auprès de pêcheurs au Mali. Ces derniers ont quitté le delta intérieur du fleuve Niger pour aller occuper les berges d'une retenue d'eau à Sélingué. Elle se trouve à quelques 1000 kilomètres de leur zone d'origine, au sud du pays et non loin des frontières avec la Guinée et la Côte d'Ivoire. Dans un premier temps, au début des années soixante-dix, ces migrants s'apparentent à des réfugiés climatiques, car les grandes sécheresses de la zone sahélienne, où ils résidaient, ont raréfié les réserves halieutiques. Mais la mise en eau de cette retenue en 1980 génère de nouvelles opportunités de pêche, et très vite de nombreux hameaux de pêcheurs itinérants vont s'établir sur les rives. Par la suite, les réseaux vont se complexifier à partir de ces lieux, les pêcheurs quittant Sélingué pour la Côte d'Ivoire, le Burkina Faso ou encore le Ghana, ou pour l'aval du fleuve. Togola montre comment ces migrations permettent aux jeunes gens d'accéder non seulement à un revenu mais de s'autonomiser par rapport aux adultes de la famille. Quoiqu'il en soit le lien avec le village d'origine demeure vivace. Il est matérialisé par des retours visant en particulier à y réactualiser les relations d'alliances matrimoniales.

- Pierre Cissé montre comment le Cameroun est devenu une destination pour les migrants maliens au tournant des années quatre-vingt avec la crise économique. Ainsi des Maliens émigrés au Tchad ou au Niger vont se réorienter vers le Cameroun, ouvrant la voie à d'autres compatriotes. Cissé décrit un contexte original, celui de l'occupation par les Maliens de niches d'emplois délaissés par les autochtones et la constitution de spécialisations, de type "ethnic bizness". Les migrants maliens réutilisent en effet des savoir-faire acquis avant ou pendant la migration, en matière de commerce (bétail ou friperie), ou acceptent de s'employer sur des métiers socialement dévalorisés dans leur propre contexte social de départ, comme les métiers de la forge. D'autre part, la description des relations avec les autochtones montre également comment les arrangements entre les uns et les autres, voire les tensions liées à des conflits d'intérêt, s'organisent progressivement. Enfin, malgré la récente aggravation du contexte économique en Afrique, le retour au village demeure difficile à envisager, tant les spécialisations professionnelles acquises dans la migration ne trouveraient pas à s'exercer au pays. 
Babacar N'Dong décrit l'émigration de communautés Bassari du Sénégal et les changements sociaux et culturels importants qu'elle entraîne. Ces migrations sont d'abord saisonnières : les Bassari, qui travaillent en ville durant la contre-saison, reviennent au village pour la période agricole et s'employant. Progressivement, ces communautés demeurées longtemps assez isolées, vont s'ouvrir au reste du pays : avec le développement du tourisme dans leur région mais également avec l'installation durable de Bassari en ville. Ainsi le mouvement est double. Dans les villages d'origine, le tourisme à la recherche d'exotisme tend à folkloriser certaines pratiques rituelles des Bassari, comme la sortie des masques ou les cérémonies initiatiques destinées aux jeunes : à l'instar de ce qui a été observé au Mali, au pays Dogon. Mais dans l'espace urbain les processus sont autres. Les Bassari y ont été scolarisés et plus généralement l'influence des missions chrétiennes s'est fait sentir. Aussi certaines pratiques culturelles sont maintenues pour affirmer une identité collective, mais en quelque sorte vidées de leur contenu symbolique et en tous cas extraites du rituel complexe et long qui les entourait. N'Dong, indique en conclusion que ces changements sont surtout liés au fait que les pratiques abandonnées ne correspondaient plus au vécu social et culturel dans l'espace urbain. Il plaide néanmoins pour une société plurielle et prend l'image du caméléon, capable de s'adapter à des environnements différents.

- Dorte Thorsen expose les stratégies migratoires de jeunes burkinabé, de la campagne vers les villes, Abidjan en Côte d'Ivoire et Ouagadougou au Burkina. Elle compare ces deux contextes et la façon dont ces jeunes s'insèrent dans une chaîne de réseaux familiaux pour émigrer. Elle montre que, malgré les événements dramatiques de Côte d'Ivoire, relatés plus bas par Mahamadou Zongo, les migrants burkinabè n'ont pas cessé d'émigrer vers ce pays, tant les revenus au pays d'origine sont faibles et précaire. L'article est focalisé sur les jeunes, qui s'emploient auprès de membres plus âgés de leur famille dans les différents lieux d'immigration. La situation est alors contrastée entre Ouagadougou, où ces jeunes sont pris en charge mais ne reçoivent qu'une rémunération dérisoire voire inexistante, et Abidjan où le salaire est la règle. Dans les deux cas, les rapports intergénérationnels se transforment et la famille élargie, qui pourrait sembler comme éclatée par la migration de ses membres les plus jeunes, constitue au contraire une ressource indispensable pour les migrants.

- Bruce Whitehouse décrit la situation précaire qui est celle des migrants originaires d'Afrique de l'Ouest établis à Brazzaville. Le contexte de crise économique au Congo accentue en effet les tensions entre émigrés et congolais, ces derniers reprochant aux immigrés leur apparent enrichissement dans le commerce. Les immigrés de leur côté vivent un harcèlement quotidien de la part des forces de police, voire même de membres de la population congolaise. Cette situation où se développe la 
xénophobie reflète principalement la déliquescence de l'État de droit, qui n'est plus à même de réguler les tensions sociales.

- Mahamadou Zongo décrit les conditions du départ de Côte d'Ivoire d'émigrés burkinabé et de leur réinstallation au Burkina. Au début des années deux-mille en effet, la guerre civile en Côte d'Ivoire va entraîner le rapatriement rapide de nombreux émigrés, causant une forte pression démographique dans les villes frontalières où ils se dirigent. Ces migrants de retour vont devoir cohabiter de façon durable non seulement avec les autochtones, mais également avec des migrants de l'intérieur, Burkinabé venus s'installer dans ces régions pour y cultiver le coton. D'autres tensions vont succéder à celles qui avaient provoqué la fuite précipitée : mis en cause comme "non nationaux" et soupçonnés d'appuyer la rébellion en Côte d'Ivoire, ils sont considérés comme des "nationaux douteux", affublés du sobriquet de "fuyards" par leurs compatriotes. Avec la normalisation en Côte d'Ivoire, certains de ces rapatriés vont y retourner, mais en gardant les pieds des deux côtés de la frontière.

- Dans un entretien avec la rédaction de la revue, Michel Agier présente les grandes lignes de son récent ouvrage ${ }^{(4)}$ sur les réfugiés en Afrique, issu d'une enquête de sept années dans les camps. Il s'intéresse à ces réfugiés de l'intérieur, chassés de leur région par les conflits ou les guerres civiles, qui se retrouvent pris en charge par les organisations humanitaires. Il décrit comment la perception des réfugiés s'est transformée au fil des dernières décennies, le réfugié perdant progressivement le statut tel que définit par les Nations Unies, pour celui de déplacé pris en charge par les dispositifs de gestion de crises, maintenu et contrôlé localement.

- Dominique Vidal montre comment les Mozambicains émigrant en Afrique du Sud ont dû s'adapter aux bouleversements de l'après apartheid. Le régime de l'apartheid avait privilégié le recrutement des immigrés, et les Mozambicains constituaient une partie importante des travailleurs des mines. La migration est depuis une référence important de la société mozambicaine tant au plan social qu'économique. Avec les années quatre-vingt et la guerre civile au Mozambique, puis l'arrivée au pouvoir de Nelson Mandela en 1994, le contexte migratoire va considérablement changer. D'une part le secteur minier va s'ouvrir aux travailleurs nationaux ; l'Afrique du Sud va d'autre part adopter une législation de plus en plus restrictive. Ne trouvant plus à s'employer dans les mines, les Mozambicains vont s'orienter vers le secteur informel avec le bâtiment, la restauration, etc. Ils sont alors confrontés à la xénophobie des nationaux, et développent des stratégies d'invisibilité afin de préserver leur statut et leurs activités précaires.

- Yoon Jung Park examine la migration chinoise en Afrique, qui débute dès les Indépendances avec l'envoi par le régime maoiste de travailleurs chinois autour des nombreux chantiers que la coopération chinoise mettait en place. Si cette première phase s'inscrivait dans l'idéologie du régime, la période actuelle répond à des impératifs 
économiques et la migration chinoise est devenue visible depuis quelques années du nord au sud de l'Afrique subsaharienne. Cette migration s'inscrit bien entendu dans la montée en puissance des relations économiques entre l'Afrique noire et la Chine. L'auteur décrit une situation de grande mobilité, caractérisée par le fait que les déplacements vers l'Afrique peuvent également être une étape vers les pays occidentaux, voire l'inverse. Les activités professionnelles sont également très variées : petits entrepreneurs, cadres du BTP, commerçants, etc. La capacité à importer sur le continent africain des produits- certes souvent critiqués pour leur mauvaise qualité - à des prix accessibles aux bas revenus africains constitue un de leurs atouts commerciaux. Finalement, ces immigrés organisent leur mobilité en fonction des nouveaux marchés qu'ils pourront ouvrir.

- Amélie Barbey rend compte de l'héritage encore actuel de la situation coloniale aux Comores, comme facteur d'émigration vers Madagascar, la Réunion ou Mayotte, seule île de l'archipel demeurée française lors de l'indépendance en 1974. Détenteurs de la nationalité française, les Mahorais, peuvent circuler librement vers La Réunion, comme vers d'autres départements français, où ils bénéficient alors des droits sociaux, tandis que les Comoriens sont attirés vers cette île constituée comme étape pour une destination plus lointaine, l'Europe et plus particulièrement la France.

- Enfin, comme pour rappeler que les migrations sont également intercontinentales, Aurelia Wa Kbawe-Segatti examine la politique européenne d'immigration et questionne l'intérêt qu'elle représente pour les pays africains. L'auteur rappelle que la volonté politique largement dominante en Europe est celle de la maîtrise des flux migratoires, avec, depuis 1997, une tendance forte à inciter les États d'origine au contrôle des mouvements migratoires à leurs frontières. Cette incitation se traduit par la mise à disposition de dispositifs de surveillance ainsi que par la signature d'accords de réadmission permettant aux pays européens de renvoyer des immigrés illégaux dans les pays de transit. L'auteur en conclut que l'Union Européenne vise à externaliser la gestion des migrations internationales, conditionnant pour partie la coopération avec les États au sud de la Méditerranée à la bonne volonté de ces derniers.

Pour conclure cette présentation, il convient de souligner rapidement quelques remarques qui se dégagent, avec plus ou moins d'intensité, de l'ensemble de ces articles. D'abord, les migrations sont dirigées vers des bassins d'emplois où les migrants savent pouvoir être mieux rémunérés que dans leur pays d'origine. Finalement, ce n'est pas la misère qui chasse les individus de leurs pays : bien plus nombreux sont leurs compatriotes qui y demeurent, certes dans une situation souvent de grande pauvreté. Mais c'est bien la demande en force de travail ou l'existence de niches d'emplois délaissées par les autochtones qui génère l'immigration. Dans cette situation la capacité d'adaptation, d'information et de l'éventuelle réorganisation des des- 
tinations dont font preuve les émigrés, sont des compétences nécessaires dans l'aventure de la migration.

Ensuite, on note que les migrations de travail, lorsqu'elles s'inscrivent dans la durée, supposent la mise en place de relais locaux permettant de s'intégrer au mieux dans le pays d'accueil. Les réseaux familiaux, dont l'importance est décrite par plusieurs auteurs de ce dossier, fonctionnent alors comme autant de ressources, mais des ressources réaménagées en fonction de la situation. Il n'y a dès lors rien d'étonnant à ce que des "routes" et des destinations soient partagées par des groupes de même origine et privilégiées par ces migrants tandis que d'autres sont inaccessibles. Il n'est pas non plus indifférent de ce point de vue que les migrations vers telle ou telle destination soient le fait des ressortissants de telle ou telle région du pays de départ, accessibles aux uns mais peu ou pas ouvertes aux autres.

Enfin, il faut relever combien l'immigration interroge les identités nationales encore récentes sur le continent africain. Certes, de nombreux accords sous-régionaux autorisent la libre circulation des personnes. Et certes aussi, les carences de l'État Civil et des administrations concernées contribuent à rendre floues les appartenances. Dans cette logique, les situations de crise économique exacerbent parfois la xénophobie, mais les immigrés, à la fois dans et hors la cité, pour reprendre la formule de Simmel à propos de la figure de l'étranger, constituent avant tout un révélateur des tensions et de l'état de la société d'installation.

Toutes les discussions actuelles sur la mobilité, les migrations de masses, les flux culturels transnationaux et les concepts d'espace, de peuple et de culture ne sont en fait des préoccupations ni nouvelles ni spécifiques au continent africain. Aux ÉtatsUnis, les travaux pionniers de l'Université de Chicago, fin $\mathrm{XIX}^{\mathrm{e}}$ et début $\mathrm{XX}^{\mathrm{e}(5)}$ montrent le caractère historique global de l'urbanisation et des mouvements de peuples ruraux vers les centres urbains. Ces études montrent comment la circulation générale de la population sur la face du globe terrestre plonge continuellement les individus dans des systèmes sociaux dans lesquels leurs places sont en permanence à construire et souvent mises en cause. Ces différentes caractéristiques se rencontrent finalement dans chaque migration particulière. Il y a là de quoi méditer concernant le contexte européen.

\section{Notes}

1. http://webnet.oecd.org/WBOS/index.aspx? lang=fr

2. Utilisant des données un peu moins récentes (2000) mais différentes et peut-être plus complètes, Cris Beauchemin et David Lessault (Population et sociétés, janvier 2009) estiment à $4 \%$ la part des sub-sahariens pour l'ensemble des immigrés installés dans un pays de l'OCDE. Quoiqu'il en soit, le constat est le même : entre 2 et 4 \%, il s'agit bien de petits effectifs.

3. Université d'Oxford, 2008.

4. Gérer les indésirables. Des camps de réfugiés au gouvernement humanitaire, Paris, Flammarion, 2008.

5. Avec, en particulier, la sortie en 1927 du livre de William Thomas et Florian Znaniecki : The Polish Peasant in Europe and America. 